\title{
EDITORIAL Hidden eating disorders and the treatment gap
}

\author{
Transtornos alimentares ocultos e disparidades de tratamento \\ Phillipa Hay \\ https://orcid.org/0000-0003-0296-6856
}

The American Psychiatric Association' Diagnostic and Statistical Manual for Mental Disorders 2013 fifth edition (DSM-5') and the World Health Organisation' International Classification of Diseases 2019 eleventh edition (ICD-1 12) introduced two new feeding and eating disorders, namely Binge Eating Disorder (BED) and Avoidant/Restrictive Food Intake Disorder (ARFID). Neither are characterised by body image disturbance - a notable departure from the classic disorders, anorexia nervosa and bulimia nervosa. Two papers in this issue present reports on $\mathrm{BED}^{3}$ and $\mathrm{ARFID}^{4}$. The papers provide diverse and important new information and perspectives on the contemporary understanding of Eating Disorders. They reach from the community to the bedside and point to the importance of translational research.

The analytic epidemiologic study by de Oliveira et al. ${ }^{3}$ reported very high rates of restricted dieting (including fasting) and binge eating in university students. This is consistent with other international research which has found similarly alarming proportions of eating disorder symptoms in general population samples. These symptoms were also associated with a high body mass index (BMI), binge eating and, in particular, with poorer food choices ${ }^{3}$. This association between problems of weight and of eating disorders is reflected in the known greater increase in both problems when they co-occur ${ }^{6}$. The challenge for the future is how to develop appropriate interventions to prevent and to manage both problems.

The second paper was a case study ${ }^{4}$ that highlighted the newly described feeding and eating disorder, ARFID. ARFID is distinctive in that a fear of weight gain or body image disturbance is not merely not required, it is proscribed. The case is important as the misdiagnosis of anorexia nervosa lead to a delay in instituting appropriate therapy to address the anxiety around fear of swallowing.

Both the papers thus highlight potentially severe physical health impacts of BED and ARFID, at respective "ends" of the BMI spectrum. Because of their recent recognition both may be mistaken for other problems or missed. The high prevalence of BED in particular is a concern as it is likely many people with BED do not realise they have a treatable eating disorder. Consequently, there is a large treatment gap with most people presenting for help over a decade after onset of symptoms or not presenting at all?.

The treatment gap had been extensively documented around the globe with many explanations proposed. These range from issues for people with disorders, such as poor access to care, poor eating disorder mental health literacy in those with problems and health care professionals, and pervasive stigma at all levels ${ }^{7}$. A recent study ${ }^{8}$ attempted to understand mechanisms behind the treatment gap and thus possible solutions. In a large general population study participants were asked a series of questions regarding their health care use and specific topics discussed during contemporaneous general practice consultations. In people with a

1 Western Sydney University, School of Medicine, Translational Health Research Institute (THRI), Campbelltown, Australia.

Received in 
DSM-5 or ICD-11 diagnosis of bulimia nervosa, BED or Other Specified Feeding or Eating Disorder, there was a high frequency of general practice consultations but not of mental health consultations (confirming the "treatment gap"). If the person's mental health was discussed, then there was an increased likelihood of that person attending a mental health specialist for care. However, this did not occur if the topic "dieting or eating" was discussed. Thus the treatment gap may be in part due to family doctors not recognising the eating disorders and/or addressing eating and weight concerns with weight loss/management advice rather than eating disorder informed treatments.

To close the treatment gap there needs to be greater awareness amongst doctors and others to consider that people presenting with eating and weight concerns may well have a treatable mental health disorder and/or to screen for such routinely. Action needs to be taken now. Hidden eating disorders are not new ${ }^{9}$, eating and weight disorders are on the rise ${ }^{5,6}$, new eating disorders are recognised, and the public health impact and burden on all services at all levels will be increasing.

\section{REFERENCES}

1. American Psychiatric Association. Diagnostic and statistical manual for mental disorders. 5th ed. Washington, DC: American Psychiatric Association; 2013.

2. World Health Organisation. International Classification of Diseases (ICD-11). Geneva: World Health Organization; 2018

3. de Oliveira J, Figueiredo LS, Cordas T. Prevalence of Eating Disorders risk behavior and "Low-carb" diet in university students. J Bras Psiquiatr. 2019;68(4):183-90.

4. Soffritti E, Passos B, Rodrigues D, Freitas S, Nazar B. Adult avoidant/restrictive food intake disorder: a case report. J Bras Psiquiatr. 2019;68(4):252-7.

5. Galmiche M, Déchelotte P, Lambert G, Tavolacci MP. Prevalence of eating disorders over the 2000-2018 period: a systematic literature review. Am I Clin Nutr. 2019;109(5):1402-13.

6. da Luz FQ, Sainsbury A, Mannan H, Touyz S, Mitchison D, Hay P. Prevalence of obesity and comorbid eating disorder behaviors in South Australia from 1995 to 2015. Int J Obes (Lond). 2017;41(7):1148-53.

7. Hart LM, Granillo MT, Jorm AF, Paxton SJ. Unmet need for treatment in the eating disorders: a systematic review of eating disorder specific treatment seeking among community cases. Clin Psychol Rev. 2011;31(5):727-35.

8. Hay P, Ghabrial B, Mannan H, Conti J, Gonzalez-Chica D, Stocks N, et al. General practitioner and mental healthcare use in a community sample of people with diagnostic threshold symptoms of bulimia nervosa, binge-eating disorder, and other eating disorders. Int J Eat Disord. 2019

9. Mond JM, Myers TC, Crosby RD, Hay PJ, Mitchell JE. Bulimic eating disorders in primary care: hidden morbidity still? J Clin Psychol Med Settings. 2010;17(1):56-63. 